\title{
Fish Bone Perforation Mimicking Acute Appendicitis
}

\author{
Alex M Almoudaris ${ }^{\mathrm{a}}$, Andre Chow ${ }^{\mathrm{a}}$, Sashin Kaneria ${ }^{\mathrm{a}}$, \\ Zainab Jiyad ${ }^{a}$, Dimitri J Hadjiminas ${ }^{\mathrm{a}, \mathrm{b}}$
}

\begin{abstract}
Fish bone ingestion is relatively common, however, resultant perforation of the small bowel is fortunately rare. We present a case of a 48-year-old gentleman who attended our emergency department complaining of severe, unrelenting right lower abdominal pain for the preceding five hours. CT scanning suggested a perforated appendix however at operation the appendix was normal. Several loops of small bowel walling off an inter-loop abscess and a fishbone were discovered in the washout fluid. A diagnosis of small bowel fish bone perforation was made intra-operatively and the patient subsequently made a full recovery without the need for bowel resection. We present this as a rare case of small bowel perforation by a fish bone where the site of perforation was unidentifiable but was treated successfully without the need for bowel resection.
\end{abstract}

Keywords: Fishbone; Perforation; Appendix; Bowel

\section{Introduction}

Fish bone ingestion is very common however resultant bowel perforation is rare. Rarer still is managing such a complication without the need for bowel resection. This case reflects upon the importance of history taking, judicious examination of pre-operative imaging and uniquely where perforation is

\footnotetext{
Manuscript accepted for publication Feb 24, 2011

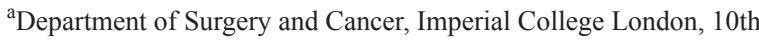
Floor, QEQM Building, St Mary's Hospital Campus, Praed Street, London, W2 1NY, United Kingdom

${ }^{\mathrm{b}}$ Corresponding author: Consultant General, Breast and Endocrine Surgeon, St Mary's Hospital Campus, Imperial College London, London, W2 1NY, United Kingdom.

E-mail: dimitri.hadjiminas@imperial.ac.uk
}

doi: $10.4021 / \mathrm{jmc} 151 \mathrm{w}$ suspected but a site unidentifiable intra-operatively due to the risk of causing further harm, such perforation can be successfully managed without bowel resection.

\section{Case Report}

A 48-year-old Caucasian gentleman presented to the Emergency Department complaining of lower right abdominal pain. He described this as being constant since coming on suddenly five hours previously. He felt feverish, but had no history of nausea or vomiting. He had opened his bowels normally prior to the onset of pain. His past medical history included alcoholism for the past seven years after the unexpected death of his young son, drinking approximately one litre of whisky per day. He was diagnosed with gallstones two years previously but had been asymptomatic.

On examination the patient was flushed and sweaty. He was febrile with a temperature of $38^{\circ} \mathrm{C}$ and tachycardic with a heart rate of 105 beats per minute. He had localised peritonism in the right iliac fossa. There was the clinical impression of a mass in the right lower zone. His urine and rectal examinations were unremarkable. His plain radiographs demonstrated no free air or radiographic evidence of bowel obstruction.

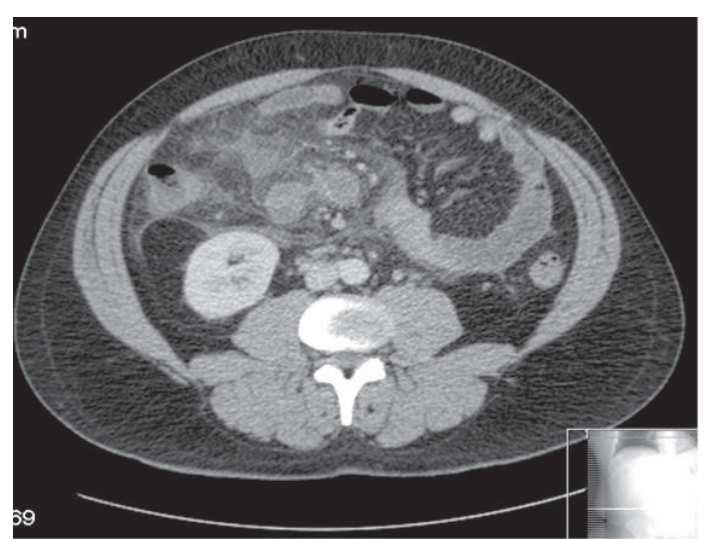

Figure 1. This is an axial section from the CT scan demonstrating the large amount of inflammatory change within the right iliac fossa. 


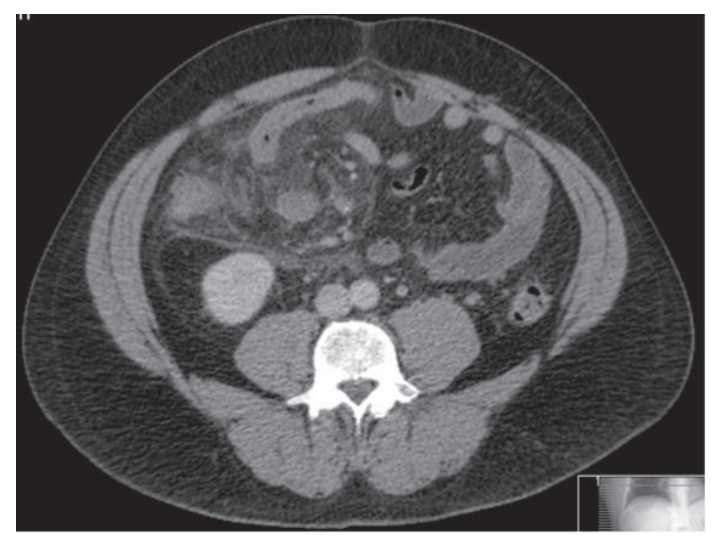

Figure 2. This is an axial section from the CT scan demonstrating a complex abscess with a loop of small bowel entering it.

His blood tests showed both a raised white cell count of 10.6 and a raised CRP of 109 with a normal amylase of 31 . His liver function tests demonstrated a raised bilirubin at 42 with an ALP of 110 and ALT 37. His arterial blood gas was unremarkable.

A CT scan was requested to investigate the nature of the mass and it demonstrated significant inflammatory change around the right iliac fossa (Fig. 1) and the region of the caecum. The appendix was not identifiable on the CT scan (Fig. 2). A presumptive diagnosis of a perforated appendix was made and the patient proceeded to diagnostic laparoscopy and laparotomy.

Under anaesthetic, on examination a mass could easily be felt in the right iliac fossa. At laparoscopy there was free pus in the pelvis and several small bowel loops fixed in the right abdominal area walling off a large abscess cavity. The appendix could not be visualised. Conversion to laparotomy was performed. By careful dissection the appendix, which appeared normal macroscopically, was identified and removed in the standard fashion. A full laparotomy was performed and all the solid organs appeared normal as did the duodenum. There was no free faeces in the abdomen. Three loops of distal ileum walling off an inter- loop abscess in the right lower abdominal compartment were separated. During washing out of the pus in this area a $3 \mathrm{~cm}$ fishbone was found floating in the fluid (Fig. 3).

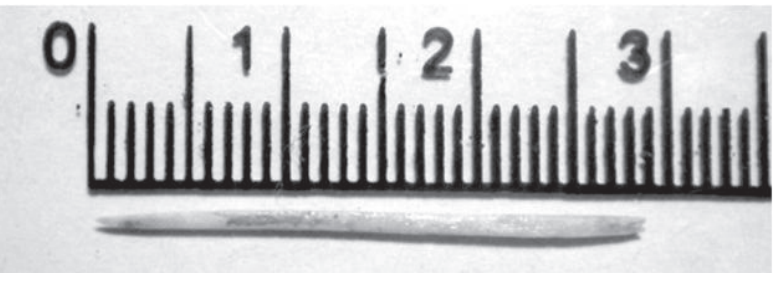

Figure 3. This is a photo of the fish bone discovered intra-operatively, the scale is in centimetres.

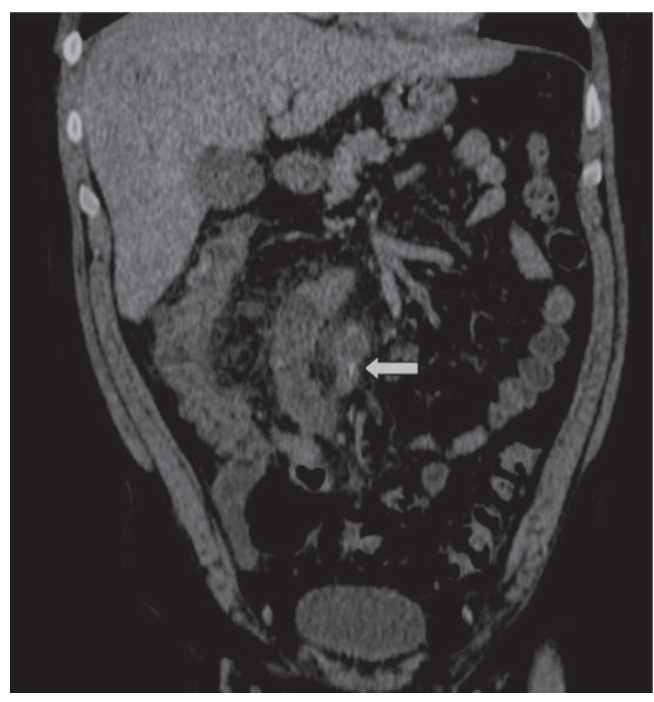

Figure 4. This is a coronal reconstruction from the CT scan that in retrospect demonstrates the fish bone within a loop of small bowel-distal ileum. It is highlighted by the white arrow.

An intraoperative diagnosis of fish bone perforation was made. After a thorough and careful re-examination of the entire bowel no definite site of perforation could be identified. The decision was made to close the abdomen following copious washout, and to treat the patient with intravenous antibiotics.

The patient made a rapid recovery and was discharged after a full course of intravenous antibiotics. On questioning the patient admitted to eating fish two nights before presenting to the ED. A review of the pre-operative CT with coronal reconstruction (Fig. 4) clearly demonstrates the fish bone on the coronal plane reconstruction.

\section{Discussion}

Any insoluble ingested foreign body may potentially cause bowel perforation, however the most commonly unintentionally ingested foreign bodies are fish bones, toothpicks, chicken bones and fragments of bone [1]. Perforation may occur at any site along the gastrointestinal tract with varying clinical presentations. However it must be noted that most ingested foreign bodies pass spontaneously through the gastrointestinal tract without impaction or perforation or need for surgical intervention.

In certain populations intentional foreign body ingestion is commonplace. In the largest review of ingested foreign bodies to date by Velitchkov et al of 542 patients from 1973 to 1993 in Bulgaria the foreign bodies ingested that caused bowel perforation included spoon handles, metal wire and metal pins [2]. However, almost $70 \%$ of the patients were jail inmates and over $20 \%$ had psychiatric conditions, therefore caution is required when interpreting these data when 
considering the population as a whole. The foreign bodies reported in this study reflect the availability of such items in prisons and the fact they are radio-opaque on plain radiographs.

Preoperative diagnosis is very difficult due to many factors. Goh et al. in their paper outlining the use of CT scanning in the diagnosis of fish bone perforation demonstrated this. From 1996-2003 22 patients were identified that had presented with bowel perforation due to foreign body ingestion- of these, 15 were due to fish bones. Seven had had preoperative CT scans. Furthermore in only 5 of the 7 scans was a fishbone perforation suspected pre-operatively. Reasons given included the poor opacification of certain types of fish bone, lack of observer awareness and more technical aspects such as too large a CT slice thickness, movement artifact, the use of oral and intravenous contrast material and orientation of a FB with respect to an axial CT scan can all make identifying fish bones extremely difficult on CT scans [3].

The wide variety of potential clinical presentations is reflected in the literature with fish and chicken bone perforations being mistaken preoperatively for numerous conditions including acute appendicitis (as in our case) and perforated diverticulitis [4]. At the other end of the spectrum colovesical and colorectal fistulas have been reported as being caused by ingested chicken bones discovered at the time of surgery $[5,6]$.

Fortunately fish bone perforation is rare, however in the estimated $1 \%$ of all ingested foreign bodies that do cause bowel perforation the most common locations for perforation of the lower gastrointestinal tract are in the ileocecal and rectosigmoid regions [1]. It is thought this occurs due to the change in direction of the intraluminal contents at these sites and the fact that at these points the colon is relatively fixed and less accommodating to intraluminal contents changing direction as opposed to the more mobile mesentery of the small bowel.

The literature with respect to foreign body bowel perforation states the site of perforation is usually identified and some form of direct closure or bowel resection is performed $[1,7]$. In our case the site of perforation was not identified despite a thorough examination. Furthermore there was only localised inflammatory change in the region of the distal ileum without evidence of more generalised peritonitis. Our patient was managed with intravenous broad-spectrum antibiotics for one week to good effect. It is indeed fortunate that the fish bone was identified during the washout however this case demonstrates that it is not always necessary to identify the site of perforation if a foreign body is discovered intraoperatively. It is likely that given the small size and profile of the fishbone in this case, that the initial perforation may have sealed spontaneously.

Fish bone perforation is a previously well-documented cause of gastrointestinal perforation [8,9]. However, to our knowledge, fish bone perforation masquerading as acute ap- pendicitis with no definite site of perforation being identified is novel. It is likely that the perforation had occurred in the distal ileum with the fibrinous reactive change involving the caecum leading to the clinical signs and the preoperative imaging suggestive of perforated appendicitis.

The appendix histology indeed came back as microscopically normal however it did show evidence of reactive inflammatory change.

\section{Conclusion}

Fish bone perforation of the small bowel is a rare but well recognised entity. We have presented a case where the site of perforation could not be identified intra-operatively, however, the patient made a swift and full recovery with intravenous antibiotics and without the need for bowel resection. This case demonstrates that fish bone perforations can be managed without the need for bowel resection following a full laparoscopy or laparotomy to exclude the presence of a macroscopic perforation.

It must be remembered however that ingested foreign bodies causing gastrointestinal perforation still remains fortunately rare. Most ingested foreign bodies pass through the gastrointestinal within 1 week and are uneventfully passed [10].

\section{Conflict of Interest}

None declared

\section{Financial Interest}

Non declared

\section{References}

1. Pinero Madrona A, Fernandez Hernandez JA, Carrasco Prats M, Riquelme Riquelme J, Parrila Paricio P. Intestinal perforation by foreign bodies. Eur J Surg. 2000;166(4):307-309.

2. Velitchkov NG, Grigorov GI, Losanoff JE, Kjossev KT. Ingested foreign bodies of the gastrointestinal tract: retrospective analysis of 542 cases. World J Surg. 1996;20(8):1001-1005.

3. Goh BK, Tan YM, Lin SE, Chow PK, Cheah FK, Ooi LL, Wong WK. CT in the preoperative diagnosis of fish bone perforation of the gastrointestinal tract. AJR Am J Roentgenol. 2006;187(3):710-714.

4. Bhatia R, Deane AJ, Landham P, Schulte KM. An unusual case of bowel perforation due to fish fin ingestion. Int J Clin Pract. 2006;60(2):229-231. 
5. Khan MS, Bryson C, O'Brien A, Mackle EJ. Colovesical fistula caused by chronic chicken bone perforation. Ir J Med Sci. 1996;165(1):51-52.

6. Read TE, Jacono F, Prakash C. Coloenteric fistula from chicken-bone perforation of the sigmoid colon. Surgery. 1999;125(3):354-356.

7. Hewett PJ, Young JF. Toothpick injuries to the gastrointestinal tract. Aust N Z J Surg. 1991;61(1):35-37.
8. Lai AT, Chow TL, Lee DT, Kwok SP. Risk factors predicting the development of complications after foreign body ingestion. Br J Surg. 2003;90(12):1531-1535.

9. McCanse DE, Kurchin A, Hinshaw JR. Gastrointestinal foreign bodies. Am J Surg. 1981;142(3):335-337.

10. Ohri SK, Hutton KA, Walsh R, Desa LA, Wood CB. Foreign body perforation of the ileum. Br J Clin Pract. 1990;44(12):647-648. 